\title{
COMPARISON OF FATTY ACID CONTENT IN CAMELLIA OLEIFERA (L.) KUNTZE OIL AND OLIVE OIL BY GC
}

\author{
Chen Guo-Chen, Ye Hang*, Wang Dong-Xue, Zhang Nai-Yan and Wei Wei
}

Guangxi Forestry Research Institute (GFRI), Nanning City; Guangxi Key Laboratory of Special Nonwood Forest Cultivation \& Utilization; Guangxi Improved variety and cultivation engineering Research Center of oil-tea camellia, Guangxi province, 530002, The People's Republic of China

Key words: Camellia oleifera, Oil, Olive oil, Unsaturated fatty acids

\begin{abstract}
The comparison of fatty acid content in Camellia oleifera (L.) Kuntze oil and olive oil was done by GC. C. oleifera oil contained more C18:1n9c, C18:2n6c, C20:1 and C22:1n9 than olive oil. Olive oil did not contain C21:0 and C24:1 but more C8:0, C16:0, C16:1, C18:0, C18:3n6, C18:3n3, C20:2, C22:0, C23:0 and C24:0 than C. oleifera oil, C. oleifera oil did not contain C20:2. C. oleifera oil and olive oil are beneficial for human health for their unsaturated fatty acids content and C. oleifera oil is healthy than olive oil.
\end{abstract}

Camellia oleifera (L.) Kuntze (Fam. Theaceae) oil is often called eastern olive oil for the high contents of unsaturated fatty acids, e.g., oleic and linoleic acids which are beneficial for human health (Ma et al. 2011). In the present study fatty acid in C. oleifera oil and olive oil has been determined and compare to judge their beneficial effect as healthy food.

C. oleifera oil "Jinmu" from China and olive oil "Abrui" from Spanish were bought in Chaoshifa supermarket. The method of detection: Prepared fatty acid methyl ester by three boron fluoride method, determined the concentration of fatty acids by gas chromatography with capillary column and flame detector (National Standard of the People's Republic of China 2008).

C. oleifera oil contained more C18:1n9c, C18:2n6c, C20:1 and C22:1n9 than olive oil, especially C. oleifera oil contained C21:0 and C24:1, but it was not detected in olive oil. Olive oil contained more C8:0, C16:0, C16:1, C18:0, C18:3n6, C18:3n3, C20:2, C22:0, C23:0, C24:0 than C. oleifera oil; Olive oil contained C20:2, but it was not detected in C. oleifera oil (Figs 1-2, Table 1).

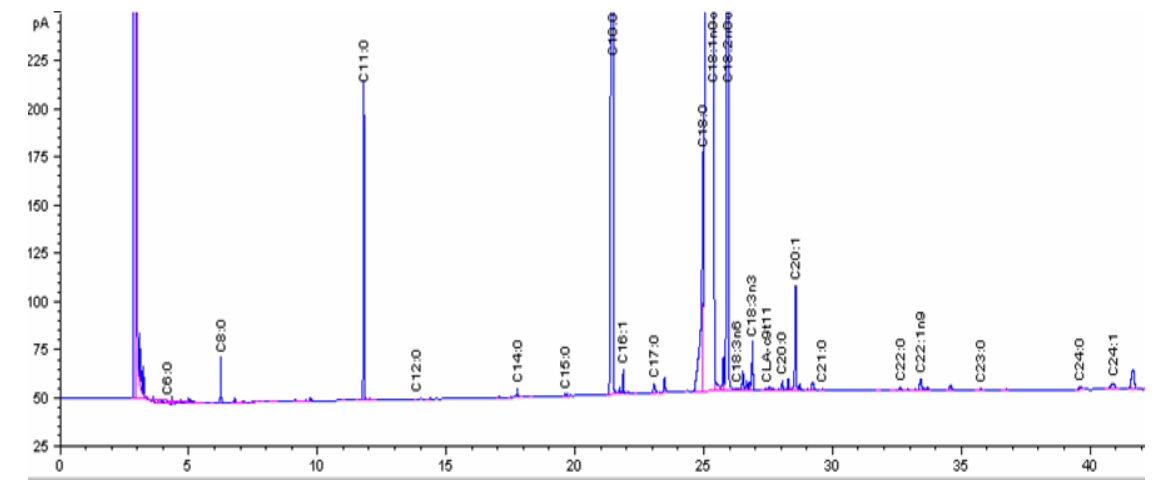

Fig. 1. Gas chromatogram of C. oleifera oil.

*Correspondence author: <ye_hang2010@163.com>. 
The results showed that $C$. oleifera oil and olive oil contained large number of unsaturated fatty acids than soybean oil and rape oil (Ma et al. 2011), which proved that Camellia oleifera oil and olive oil are beneficial for human health (Table 2).

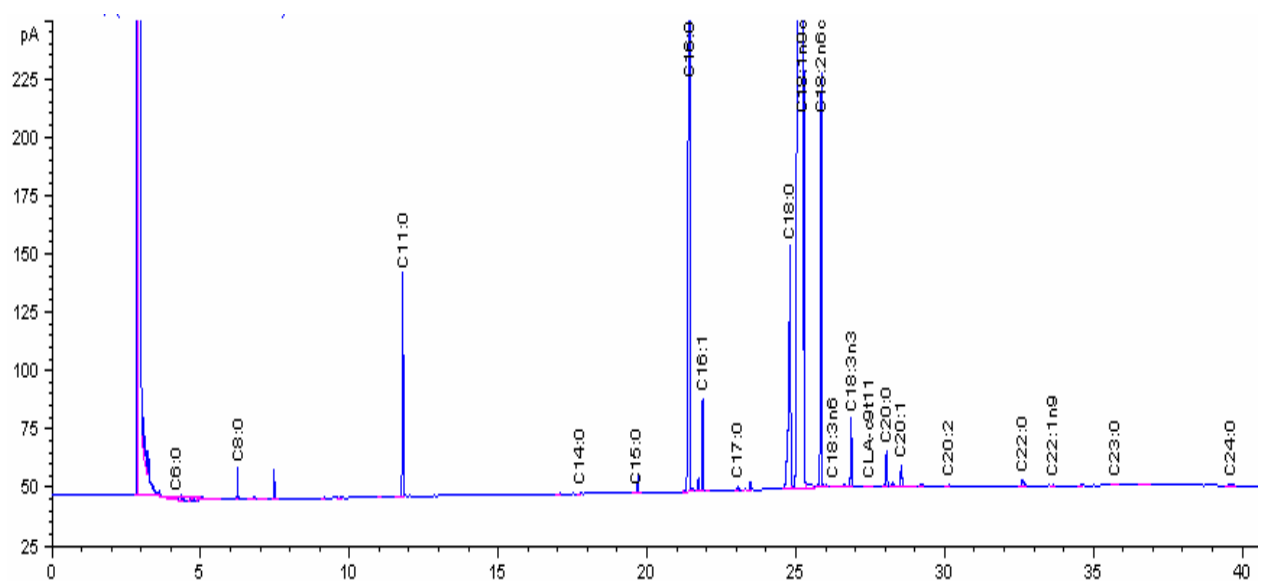

Fig. 2 Gas chromatogram of olive oil.

Table 1. The concentration of fatty acids in C. oleifera oil and olive oil (mg/g).

\begin{tabular}{lcc}
\hline Fatty acids & Olive oil & Camellia oleifera oil \\
\hline C6:0 & 0.10 & 0.05 \\
C8:0 & 1.51 & 1.05 \\
C10:0 & 0.17 & 0.15 \\
C12:0 & 0 & 0.03 \\
C14:0 & 0.11 & 0.33 \\
C15:0 & 0.04 & 0.07 \\
C16:0 & 95.25 & 74.51 \\
C16:1 & 7.07 & 0.87 \\
C17:0 & 0.42 & 0.68 \\
C18:0 & 33.85 & 20.25 \\
C18:1n9c & 729.62 & 736.42 \\
C18:2n6c & 36.89 & 71.80 \\
C18:3n6 & 0.27 & 0.03 \\
C18:3n3 & 5.45 & 2.42 \\
CLA-c9t11 & 0.08 & 0.06 \\
C20:0 $0: 12$ & 0.44 \\
C20:1 & 3.56 & 5.17 \\
C21:0 & 2.20 & 0.05 \\
C20:2 & 0 & 0 \\
C22:0 & 0.13 & 0.19 \\
C22:1n9 & 1.01 & 0.95 \\
C22:2 & 0.07 & 0 \\
C23:0 & 0 & 0.07 \\
C24:0 & 0.23 & 0.35 \\
C24:1 & 0.46 & 1.10 \\
\hline & 0 & \\
\hline
\end{tabular}


C. oleifera oil contained more C18:1n9c, C18:2n6c, C20:1 and C22:1n9 than olive oil, olive oil do not contain C21:0 and C24:1. Olive oil contained more C8:0, C16:0, C16:1, C18:0, C18:3n6, C18:3n3, C20:2, C22:0, C23:0, C24:0 than C.oleifera oil, C. oleifera oil does not contain C20:2.

Table 2. The concentration of saturated fatty acids and unsaturated fatty acids in Camellia oleifera oil and olive oil (mg/g).

\begin{tabular}{llc}
\hline Fatty acids & Olive oil & Camellia oleifera oil \\
\hline Saturated fatty acids & 136.71 & 98.22 \\
Unsaturated fatty acids & 781.78 & 818.82 \\
\hline
\end{tabular}

C. oleifera oil and olive oil contained many unsaturated fatty acids and so C. oleifera oil and olive oil may be considered beneficial for human health. As far the unsaturated fatty acids concerned C. oleifera oil is healthier than olive oil.

\section{Acknowledgements}

The supports extended by Special Research for Public Welfare in Forestry Industry(No. 201404702-05), and Guangxi Scientific Research and Technology Development (No. GKG1123004-2A) are thankfully acknowledged.

\section{References}

Ma JL, Ye H, Rui YK, Chen GC, Zhang NY 2011. Fatty acid composition of Camellia oleifera oil. J.F. Verbrauch. and Lebensmitt. 6(1): 9-12

National Standard of the People's Republic of China. Determination of fatty acid content in feed. GB/T21514-2008, Beijing: China Standards Press, 2008, 14.

Mu TN, Sun T, Wu YT, Zhao YQ 2011. Investigation of unsaturated fatty acids in three edible vegetable oils. Sci. and Technol. Cereals, Oils and Foods 19(3): 36-38. 